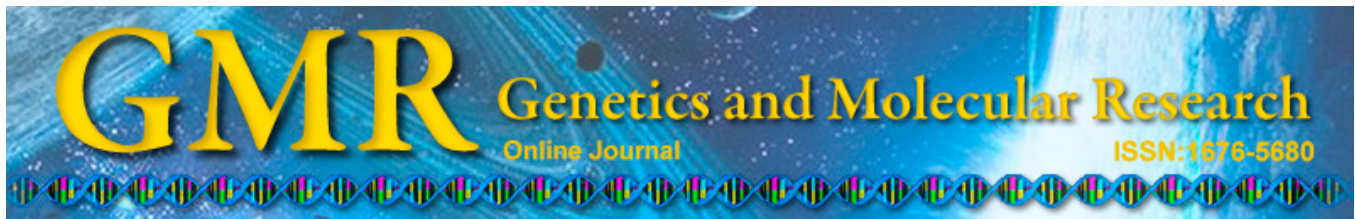

\title{
Potassium contributes to zinc stress tolerance in peach (Prunus persica) seedlings by enhancing photosynthesis and the antioxidant defense system
}

\author{
Z.Z. Song ${ }^{1,2}$, C.L. Duan ${ }^{3}$, S.L. Guo ${ }^{1,2}$, Y. Yang ${ }^{1,2}$, Y.F. Feng ${ }^{4}$, R.J. Ma ${ }^{1,2}$ and \\ M.L. Yu ${ }^{1,2}$ \\ ${ }^{1}$ Institute of Horticulture, Jiangsu Academy of Agricultural Sciences, \\ Nanjing, China \\ ${ }^{2}$ Jiangsu Key Laboratory of Horticultural Crop Genetic Improvement, \\ Nanjing, China \\ ${ }^{3}$ Jiangsu Provincial Fishery Technical Extending Center, Nanjing, China \\ ${ }^{4}$ Institute of Agricultural Resources and Environment, \\ Jiangsu Academy of Agricultural Sciences, Nanjing, China \\ Corresponding author: Z.Z. Song / M.L. Yu \\ E-mail: szhzh2000@163.com / mly1008@aliyun.com \\ Genet. Mol. Res. 14 (3): 8338-8351 (2015) \\ Received October 24, 2014 \\ Accepted April 24, 2015 \\ Published July 27, 2015 \\ DOI http://dx.doi.org/10.4238/2015.July.27.22
}

\begin{abstract}
Zinc ( $\mathrm{Zn})$ is considered to be a major industrial pollutant because excessive amounts can impair plant growth. In this paper, we found that peach 'Yoshihime' seedlings are promising $\mathrm{Zn}$ tolerant plants. However, heavy $\mathrm{Zn}$ toxicity $(2 \mathrm{mM})$ damaged plant performance by disrupting biochemical processes, including photosynthesis, proline production, and $\mathrm{K}^{+}$nutrition. Notably, elevated external $\mathrm{K}^{+}$supply $(10 \mathrm{mM})$ alleviated peach seedlings from $\mathrm{Zn}$ toxicity, evidenced by enhanced photosynthesis, antioxidant defense systems, and plant $\mathrm{K}^{+}$ nutritional status. Moreover, the transcript levels of $K U P\left(\mathrm{~K}^{+}\right.$uptake) genes involved in $\mathrm{K}^{+}$acquisition, transport, and homeostasis were
\end{abstract}


significantly upregulated following supply of sufficient $\mathrm{K}^{+}$upon $\mathrm{Zn}$ toxicity. In general, $\mathrm{K}^{+}$favorably contributes to improvements in internal $\mathrm{K}^{+}$homeostasis, via the help of $\mathrm{K}^{+}$transporters, further protecting plant photosynthesis and the antioxidative defense system. Our findings further benefit the study of the mechanisms underpinning heavy metal tolerance in woody plants.

Key words: Potassium; Photosynthesis; Antioxidant defense system; Peach; Zinc stress

\section{INTRODUCTION}

High concentrations of heavy metals can adversely affect the growth, fruit formation, and development of fruit trees (Worthington, 2001; Lombardi and Sebastiani, 2005; Foyer and Noctor, 2005). Yet, zinc ( $\mathrm{Zn}$ ) sprays at critical periods can improve the fruit quality of apple trees (Zhang et al., 2013), and foliar application of Zn contributes to soluble carbohydrate and oil contents in olive cultivars during fruit ripening (Saadati et al., 2013). However, due to its high mobility and bioavailability, $\mathrm{Zn}$ is considered to be a major industrial pollutant of both the terrestrial and aquatic environments (Barak and Helmke, 1993; Morillo et al., 2004). Excessive amounts of $\mathrm{Zn}$ cause leaf chlorosis, nutrient imbalance, and inhibition of photosynthesis at various stages, resulting in damage to plant growth (Kabata-Pendias and Pendias, 2001; Morillo et al., 2004; Street et al., 2007). Common plant seedlings, such as rice, Arabidopsis, and maize, experienced mortality under $0.5 \mathrm{mM}$ Zn stress (Song ZZ, Guo SL, Ma RJ and Yu ML, unpublished results). Despite this, certain plant species, such as Silene vulgaris and Halimione portulacoides, have evolved a tolerance to $\mathrm{Zn}$ that enables them to grow in $\mathrm{Zn}$-contaminated soils, remaining unaffected by the elevated Zn levels (Ernst et al., 2000; Cambrollé et al., 2012).

Plants suffering from adverse abiotic stresses, including heavy-metal stress, produce excess internal reactive oxygen species (ROS), such as superoxide $\left(\mathrm{O}_{2}^{-}\right)$, hydrogen peroxide $\left(\mathrm{H}_{2} \mathrm{O}_{2}\right)$, and hydroxyl radicals $\left(\cdot \mathrm{OH}^{-}\right)$, which negatively elicit oxidative stress on cellular structures and metabolism (Foyer and Noctor, 2005; Turchi et al., 2012; Singh et al., 2012; Song et al., 2014a). In particular, plants can cope with oxidative stress via the antioxidant defense system. The activity of antioxidant enzymes, i.e., superoxide dismutase (SOD), peroxidase (POD), catalase (CAT), and ascorbate peroxidase (APX), are correlated with plant tolerance to abiotic stresses (Wang et al., 2009; Huang et al., 2013). Previous reports illustrate that heavy metal toxicity results in oxidative damage to plants and that heavy metal tolerant plants possess high antioxidant enzyme activity (Turchi et al., 2012; Singh et al., 2012). In particular, proline accumulates heavily under stresses, providing the plants protection against damage by ROS, which helps to protect enzyme activities, stabilize protein synthesis machinery, or regulate cytosolic acidity (Choudhary et al., 2007).

As one of the most abundant cations in cells, potassium $\left(\mathrm{K}^{+}\right)$not only contributes to plant growth, development, and fruit quality and yield (Maathuis and Sanders, 1996; Véry and Sentenac, 2003; Demiral and Köseoglu, 2005; Hartz et al., 2005), but it also favorably improves plant tolerance to various abiotic stresses, such as drought (Li et al., 2011; Song and Su, 2013; Song et al., 2014a), salt (Li et al., 2011; Mian et al., 2011; Song et al., 2014b), and cold (Rai et al., 2008). However, studies of $\mathrm{K}^{+}$in relation to plant tolerance to heavy metals have not been undertaken to date. 
In particular, the KT/HAK/KUP family of transporters, as part of the high-affinity $\mathrm{K}^{+}$transport system, are effective in transporting external $\mathrm{K}^{+}$, via involvement in root hair elongation or cell expansion growth (Véry and Sentenac, 2003; Grabov, 2007; Alemán et al., 2011; Coskun et al., 2013). Notably, $\mathrm{K}^{+}$transporters in the grape berry (Vitis vinifera $\mathrm{L}$.) were involved in potassium accumulation in fruit (Davies et al., 2006). Since the first plant member of the KT/HAK/KUP family was identified in barley (Hordeum) (Santa-Maria et al., 1997), subsequent studies have been conducted on evolutionarily diverse organisms (mentioned in Grabov, 2007).

In previous studies, we identified 16 putative $K U P\left(\mathrm{~K}^{+}\right.$uptake) transporters in the peach genome (Song et al., 2015). In this present study, we evaluated the physiological response of peach seedlings exposed to various external $\mathrm{Zn}$ stress levels (ranging from 0.015-2 $\mathrm{mM}$ ) and subsequently estimated the capability of improved $\mathrm{Zn}$ tolerance in relation to elevated $\mathrm{K}^{+}$supply. This present study directly indicates that $\mathrm{K}^{+}$contributes to improving plant tolerance to heavy-metal stress, which provides evidence for evaluating $\mathrm{K}^{+}$for its potential role in phytoremediation of increasing heavy metal polluted waterbodies.

\section{MATERIAL AND METHODS}

\section{Plant material and culture conditions}

Peach (Prunus persica cv. 'Yoshihime') seedlings were used throughout this study. Seed kernels were washed with distilled water and germinated in soil in a greenhouse. Germinated seedlings of a similar growth status were transferred from soil to $1 / 2 \mathrm{MS}$ solution (containing $0.015 \mathrm{mM} \mathrm{Zn}^{2+}$, Murashige and Skoog, 1962), and exposed to different treatments in aerated plastic containers. Greenhouse conditions were maintained at $25^{\circ} \pm 2^{\circ} \mathrm{C}$ and $12 / 12$ $\mathrm{h}$ light/dark, with $60 \%$ relative humidity. The nutrient solution was refreshed every other day.

\section{Stress treatments}

For the $\mathrm{Zn}^{2+}$ toxicity treatments, we carried out three parallel sets of experiments using 8 peach seedlings in each. The final $\mathrm{Zn}^{2+}$ concentration was adjusted to the desired amounts of 0.015 (the control, present in 1/2 MS basic media), $0.1,0.5$, or $2 \mathrm{mM}$.

To determine the role of external $\mathrm{K}^{+}$supply in plant performance under $\mathrm{Zn}$ toxicity, three parallel sets of experiments using 8 peach seedlings each were exposed to $2 \mathrm{mM} \mathrm{ZnCl}$, supplied with $1(1 \mathrm{~K}+\mathrm{Zn}$ treatment $)$ or $10 \mathrm{mM}(10 \mathrm{~K}+\mathrm{Zn}$ treatment $) \mathrm{KCl}$, respectively. All treatments were maintained for 10 days (for phenotype assay) or 21 days (for gene expression and antioxidant enzyme activity analysis) prior to analysis.

\section{Physiological measurements}

Seedlings were rinsed in distilled water, soaked, and then weighed to obtain fresh weight. The roots were scanned with an Epson Rhizo scanner (RHIZO 2004b, Long Beach, CA, USA), and total root length and surface area were acquired using the Epson WinRHIZO software installed in the Rhizo scanner. The seedlings were separated into roots, stems, and leaves, dried in an oven at $105^{\circ} \mathrm{C}$ for $30 \mathrm{~min}$, and then baked at $70^{\circ} \mathrm{C}$ for $48 \mathrm{~h}$ to obtain the dry weight of the biomass. The dried samples were ground into fine powder and were fully di- 
gested with $\mathrm{HNO}_{3}-\mathrm{HClO}_{4}$, and $\mathrm{K}^{+}$and $\mathrm{Zn}^{2+}$ concentrations were measured using flame atomic absorption spectrometry. Photosynthetic analysis was carried out on a portable photosynthetic system LI-6400 (Li-COR, Lincoln, NE, USA) to determine net photosynthetic rate $\left(P_{N}\right)$ and stomatal conductance $\left(g_{s}\right)$ at the terminal leaflet of fully grown second leaves, as described by Kumar et al. (2006). Chlorophyll and proline determination were carried out according to the description of Song et al. (2014a). The total protein concentration of leaf samples was determined according to the method specified by Bradford (1976), and was then used immediately for antioxidant enzyme assays. SOD, POD, CAT, and APX activities, and $\mathrm{H}_{2} \mathrm{O}_{2}$ level were determined using the relevant detection kits (Jiancheng Bioengineering Institute, Nanjing, China), according to the manufacturer protocol.

\section{Isolation of candidate genes involved in the antioxidant defense system}

Sequences of genes encoding putative antioxidant enzymes used in this study were obtained by querying the protein sequences of Arabidopsis SOD (AT4G25100 and AT1G08830), POD (AT4G30170), CAT (AT1G20630), or APX (AT1G07890) against the BLAST peach genome in the Genome Database for Rosaceae. Successfully, we obtained 4 putative SOD (PpeSOD1-4), 3 POD (PpePOD1-3), 1 CAT (PpeCAT1), and 4 APX (PpeAPX1-4) encoding genes, respectively.

\section{RNA extraction and quantitative real-time PCR (qRT-PCR)}

Total RNA was extracted from leaves, stems, and roots using a Plant RNA Kit (Omega, Norcross, GA, USA) and was reverse transcribed into cDNA using a reverse transcription kit (TaKaRa, Tokyo, Japan). qRT-PCR was carried out in a 7500 Real Time PCR System (Applied Biosystems, USA). Specific primers for genes and the control gene ubiquitin in peach were designed using the NCBI/Primer-BLAST online server (listed in Table 1). PCR conditions for thermal cycling were as follows: $95^{\circ} \mathrm{C}$ for $30 \mathrm{~s}, 40$ cycles of $95^{\circ} \mathrm{C}$ for $5 \mathrm{~s}$ and $60^{\circ} \mathrm{C}$ for 30 $\mathrm{s}$. The relative expression levels of the target genes were presented after normalization to the internal control from four independent biological repeats.

\section{Statistical and graphical analysis}

Data were statistically analyzed using the SPSS 13.0 software (SPSS Chicago, IL, USA). Details are provided in both the text and figure legends. Graphs were produced using the Origin 8.0 software.

\section{RESULTS}

\section{Physiological responses of peach seedlings to external Zn stress}

Peach, as a perennial woody plant, exhibits good $\mathrm{Zn}$ tolerance and can survive in 0.5 $\mathrm{mM} \mathrm{Zn}{ }^{2+}$ stress (Figure 1). Compared to control conditions $\left(0.015 \mathrm{mM} \mathrm{Zn}^{2+}\right)$, shoot growth and root elongation of peach seedlings were inhibited after being subjected to $2 \mathrm{mM} \mathrm{Zn}$ toxicity (Figure 1a), evidenced by dramatically reduced total fresh weight, total root length, and surface area (Figure 1b-d). Notably, Zn toxicity also significantly reduced leaf chlorophyll 
Table 1. Primer pairs used for quantitative RT-PCR experiments in peach seedlings exposed to zinc toxicity.

\begin{tabular}{|c|c|c|c|}
\hline Gene & Pfam annotation & Primer (5' to $\left.3^{\prime}\right)$ & Amplicon size (bp) \\
\hline $\begin{array}{l}\text { PpeSOD1 } \\
\text { (ppa008765m) }\end{array}$ & Putative iron superoxide dismutase & $\begin{array}{l}\text { F: GAAGGGCGTTGCTGTTCTTG } \\
\text { R: GCAACCGTTGGTTGTGTCTC }\end{array}$ & 163 \\
\hline $\begin{array}{l}\text { PpeSOD2 } \\
\text { (ppa012845m) }\end{array}$ & Putative copper/zinc superoxide dismutase & $\begin{array}{l}\text { F: GAAGGGCGTTGCTGTTCTTG } \\
\text { R: GCAACCGTTGGTTGTGTCTC }\end{array}$ & 163 \\
\hline $\begin{array}{l}\text { PpeSOD3 } \\
\text { (ppa012725m) }\end{array}$ & Putative copper/zinc superoxide dismutase & $\begin{array}{l}\text { F: CCCAGATGGAGTTGCTGAGG } \\
\text { R: TCTTGCTCCTGCATTCCCAG }\end{array}$ & 160 \\
\hline $\begin{array}{l}\text { PpeSOD4 } \\
\text { (ppa009729m) }\end{array}$ & Putative copper/zinc superoxide dismutase & $\begin{array}{l}\text { F: GGCCTCTCTGTTAAGCTCCC } \\
\text { R: CACAGTTGTAGGCCCATCGT }\end{array}$ & 177 \\
\hline $\begin{array}{l}\text { PpePOD1 } \\
\text { (ppa008569m) }\end{array}$ & Putative peroxidase precursor & $\begin{array}{l}\text { F: TACGCATTGCAGCTCAGACA } \\
\text { R: CCAACTTGGTCATGGCTTGC }\end{array}$ & 193 \\
\hline $\begin{array}{l}\text { PpePOD2 } \\
(\text { ppa008489m) }\end{array}$ & Putative peroxidase precursor & $\begin{array}{l}\text { F: AGGGTTGTGATGCTTCGGTT } \\
\text { R: CCGTCCAATCTCCCCAACTC }\end{array}$ & 249 \\
\hline $\begin{array}{l}\text { PpePOD3 } \\
\text { (ppa015245m) }\end{array}$ & Putative peroxidase precursor & $\begin{array}{l}\text { F: AAATGGGGACGCAGAGAAGG } \\
\text { R: AACGACCTGAGAGGCTTGTG }\end{array}$ & 232 \\
\hline $\begin{array}{l}\text { PpeCAT1 } \\
\text { (ppa004776m) }\end{array}$ & Putative catalase protein & $\begin{array}{l}\text { F: CACATGGAAGGCTCTGGTGT } \\
\text { R: CCTTGGTAGCATGGCTGTGA }\end{array}$ & 160 \\
\hline $\begin{array}{l}\text { PpeAPXI } \\
\text { (ppa010431m) }\end{array}$ & Putative ascorbate peroxidase & $\begin{array}{l}\text { F: GGTCACACTCTGGGAAGGTG } \\
\text { R: TCAACAAGAGGGCGGAAGAC }\end{array}$ & 182 \\
\hline $\begin{array}{l}\text { PpeAPX2 } \\
\text { (ppa010426m) }\end{array}$ & Putative ascorbate peroxidase & $\begin{array}{l}\text { F: TGGATATTGCGGTCAGGCTC } \\
\text { R: GTGGCGGTTCTTGTTTGTCC }\end{array}$ & 159 \\
\hline $\begin{array}{l}\text { PpeAPX3 } \\
\text { (ppa009538m) }\end{array}$ & Putative ascorbate peroxidase & $\begin{array}{l}\text { F: TACGACGCCAAGACGAAGAC } \\
\text { R: GCTCAACTGCAACAACACCC }\end{array}$ & 181 \\
\hline $\begin{array}{l}\text { PpeAPX4 } \\
\text { (ppa009582m) }\end{array}$ & Putative ascorbate peroxidase & $\begin{array}{l}\text { F: CCAGATGCCAAACAAGGTGC } \\
\text { R: AAGGCTCATTCGTCCAAGGG }\end{array}$ & 154 \\
\hline $\begin{array}{l}\text { PpeKUPl } \\
\text { (ppa025447m) }\end{array}$ & Putative potassium transporter & $\begin{array}{l}\text { F: AGGCGTTGAAGGATGGGTTT } \\
\text { R: GGCCAAAACACAGCTTCTGG }\end{array}$ & 234 \\
\hline $\begin{array}{l}\text { PpeKUP2 } \\
\text { (ppa002879m) }\end{array}$ & Putative potassium transporter & $\begin{array}{l}\text { F: AGCGGAGGAAGGAGGTTAGT } \\
\text { R: GTGATACCCCACTTGCCACA }\end{array}$ & 165 \\
\hline $\begin{array}{l}\text { PpeKUP3 } \\
\text { (ppa001638m) }\end{array}$ & Putative potassium transporter & $\begin{array}{l}\text { F: TAGAGGAGACTCAACCGGCA } \\
\text { R: CGAGTATAAACGCCGTCCCA }\end{array}$ & 213 \\
\hline $\begin{array}{l}\text { PpeKUP4 } \\
\text { (ppa001724m) }\end{array}$ & Putative potassium transporter & $\begin{array}{l}\text { F: CCGACCCAACGAAGGAAGAA } \\
\text { R: TACGCTGAGTGCATAGCTGG }\end{array}$ & 240 \\
\hline $\begin{array}{l}\text { PpeKUP5 } \\
\text { (ppa003114m) }\end{array}$ & Putative potassium transporter & $\begin{array}{l}\text { F:TGGGTCCCCATTGCTCTTTC } \\
\text { R: CGGACCCTAACAATCCCCAG }\end{array}$ & 152 \\
\hline $\begin{array}{l}\text { PpeKUP6 } \\
\text { (ppa022818m) }\end{array}$ & Putative potassium transporter & $\begin{array}{l}\text { F: AGTCTGTGAGCTTGGTGAGC } \\
\text { R: AAGTACGCTGTGCCACTCTC }\end{array}$ & 196 \\
\hline $\begin{array}{l}\text { PpeKUP7 } \\
\text { (ppa001652m) }\end{array}$ & Putative potassium transporter & $\begin{array}{l}\text { F: GTGCATCGTCAGGTATGGGT } \\
\text { R: GATGGAGTCGCTCACACCAA }\end{array}$ & 235 \\
\hline $\begin{array}{l}\text { PpeKUP8 } \\
(\text { ppa022284m) }\end{array}$ & Putative potassium transporter & $\begin{array}{l}\text { F: AACTCCTGAAGGTGCTGTGG } \\
\text { R: TAGGTGGCCTGCAAGTTCTG }\end{array}$ & 182 \\
\hline $\begin{array}{l}\text { PpeKUP9 } \\
\text { (ppa001335m) }\end{array}$ & Putative potassium transporter & $\begin{array}{l}\text { F: GACAATCAGAGCACCGGGAA } \\
\text { R: GACACGCCGGAAAAGAAACC }\end{array}$ & 181 \\
\hline $\begin{array}{l}\text { PpeKUP10 } \\
\text { (ppa001373m) }\end{array}$ & Putative potassium transporter & $\begin{array}{l}\text { F: TTGTCCAAGGCATCCCATCC } \\
\text { R: CGGGCTATACAGCGGAACAT }\end{array}$ & 178 \\
\hline $\begin{array}{l}\text { PpeKUP11 } \\
\text { (ppa016701m) }\end{array}$ & Putative potassium transporter & $\begin{array}{l}\text { F: CGGAAAGATTGGCAACGCAT } \\
\text { R: AGAGGAAGGAAACCGCCTTG }\end{array}$ & 201 \\
\hline $\begin{array}{l}\text { PpeKUP12 } \\
\text { (ppa015062m) }\end{array}$ & Putative potassium transporter & $\begin{array}{l}\text { F: CATACCTTCCGTCCACTCCG } \\
\text { R: TCAACCCTGTCGTTGTAGCC }\end{array}$ & 159 \\
\hline $\begin{array}{l}\text { PpeKUP13 } \\
\text { (ppa001648m) }\end{array}$ & Putative potassium transporter & $\begin{array}{l}\text { F: GGCATACACTGGACAAGCCT } \\
\text { R: CCTGGCCGATGTGTGCACGA }\end{array}$ & 226 \\
\hline $\begin{array}{l}\text { PpeKUP14 } \\
\text { (ppa001603m) }\end{array}$ & Putative potassium transporter & $\begin{array}{l}\text { F: TGCCTGCGATCCATTCTGTT } \\
\text { R: CATCCGAGTCTGAACACCCC }\end{array}$ & 249 \\
\hline $\begin{array}{l}\text { PpeKUP15 } \\
\text { (ppa001626m) }\end{array}$ & Putative potassium transporter & $\begin{array}{l}\text { F: AATTGGCAATGCCTCTGGGA } \\
\text { R: GCGATCACAAGAGGAACCCA }\end{array}$ & 204 \\
\hline $\begin{array}{l}\text { PpeKUP16 } \\
\text { (ppa016662m) }\end{array}$ & Putative potassium transporter & $\begin{array}{l}\text { F: GTCAATCAAGGTGGGTGGGT } \\
\text { R: GGCGGGAAGGTTGGTGATAA }\end{array}$ & 246 \\
\hline $\begin{array}{l}\text { Ubiquitin } \\
\text { (ppa005503m) }\end{array}$ & Ubiquitin & $\begin{array}{l}\text { F: GGCTAAGATCCAGGACAAGGAG } \\
\text { R: CCACGCAGACGGAGCACCAAG }\end{array}$ & 145 \\
\hline
\end{tabular}

Primer sequences of putative $K U P$ antioxidant enzyme encoding genes were obtained using a query protein sequence of Arabidopsis KUP1 (AT2G30070), SOD (AT4G25100 and AT1G08830), POD (AT4G30170), CAT (AT1G20630) or APX (AT1G07890), respectively, against the BLAST Peach genome at Genome Database for Rosaceae. 
concentration (Figure 2a), $P_{N}$ (Figure 2b), and $g_{s}$ (Figure 2c), but enhanced leaf proline concentration (Figure 2d). Simultaneously, $\mathrm{Zn}^{2+}$ accumulation was reinforced with increasing external $\mathrm{Zn}^{2+}$ supplies, especially in the aboveground parts of the seedlings (Figure 2e). In contrast, $\mathrm{K}^{+}$accumulation was simultaneously decreased, especially in the aboveground parts of the seedlings (Figure $2 \mathrm{f}$ ).

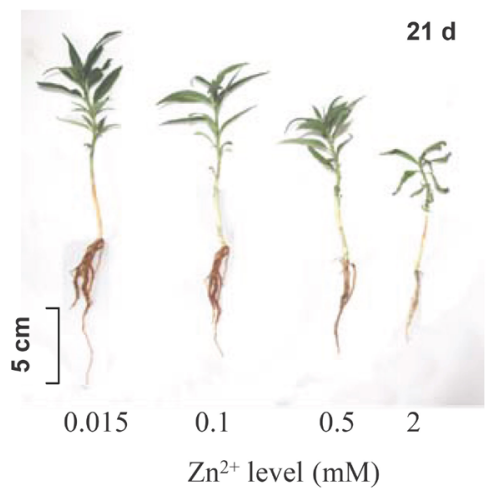

C

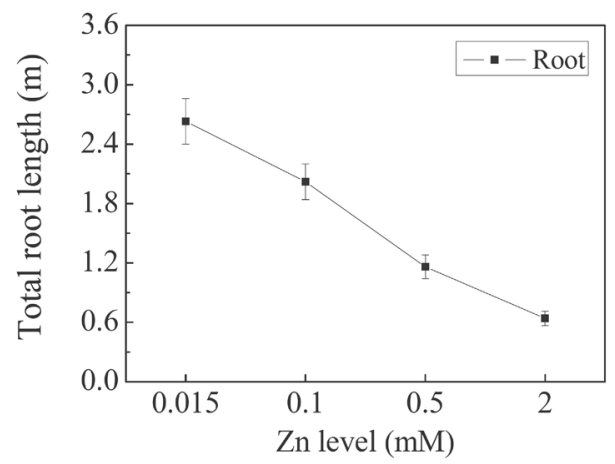

b

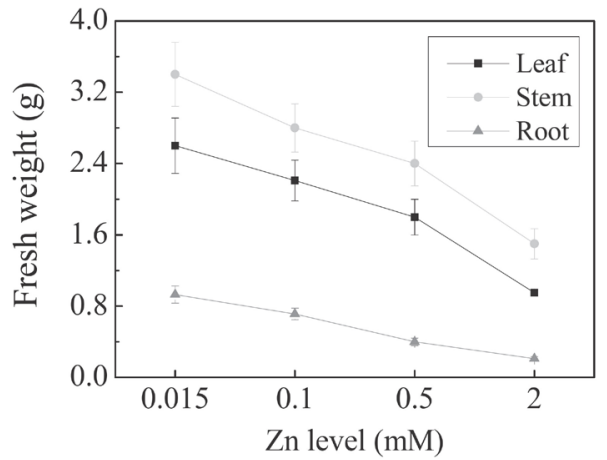

d

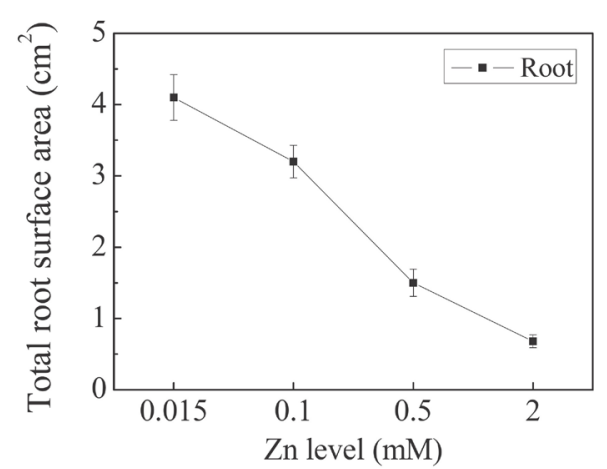

Figure 1. Growth performance of peach seedlings in response to external zinc supply. Peach seedlings were grown in a $1 / 2 \mathrm{MS}$ solution supplemented with $0.015,0.1,0.5$, or $2 \mathrm{mM} \mathrm{ZnCl}$ for 21 days before analysis. a. Phenotype; b. total fresh weight; c. total root length and surface area; d. leaf chlorophyll concentration. The values are reported as means $\pm \mathrm{SE}(\mathrm{N}=24)$, calculated from 3 sets of experiments.

\section{Elevated $\mathrm{K}^{+}$supply contributes to tolerance to zinc toxicity}

Compared with control conditions, the addition of $2 \mathrm{mM} \mathrm{Zn}^{2+}(1 \mathrm{~K}+\mathrm{Zn}$ treatment) caused severe damage (Figure 3), evidenced by reduced total fresh biomass (approximately $68 \%$ loss, $\mathrm{P}<0.01$, $t$-test), whereas the application of $10 \mathrm{mM} \mathrm{K}^{+}(10 \mathrm{~K}+\mathrm{Zn}$ treatment $)$ protected the seedlings from such damage, resulting in a significant increase in fresh biomass ( $\mathrm{P}<0.01, t$-test) and root elongation $(\mathrm{P}<0.01, t$-test), accompanied by increased chlorophyll concentration in the leaves $(0.01<\mathrm{P}<0.05, t$-test $)$, increased $g_{s}(\mathrm{P}<0.01, t$-test $)$, and increased $P_{N}\left(\mathrm{P}<0.01, t\right.$-test) (Table 2). Compared with the $1 \mathrm{~K}+\mathrm{Zn}$ treatment, elevated $\mathrm{K}^{+}$ supply $(10 \mathrm{~K}+\mathrm{Zn})$ also significantly reduced tissue $\mathrm{Zn}^{2+}$ concentration $(33 \%$ at the whole-plant 
level, $\mathrm{P}<0.01$, $t$-test) but enhanced $\mathrm{K}^{+}$accumulation $(25 \%, 0.01<\mathrm{P}<0.05, t$-test) (Table 2$)$. Moreover, elevated $\mathrm{K}^{+}$supply $(10 \mathrm{mM} \mathrm{K}$ ) significantly reduced proline concentration (approximately $30 \%, 0.01<\mathrm{P}<0.05, t$-test) under $2 \mathrm{mM} \mathrm{Zn}$ toxicity, compared to that of the 1 $\mathrm{mM} \mathrm{K} \mathrm{K}^{+}$supply (Table 2).
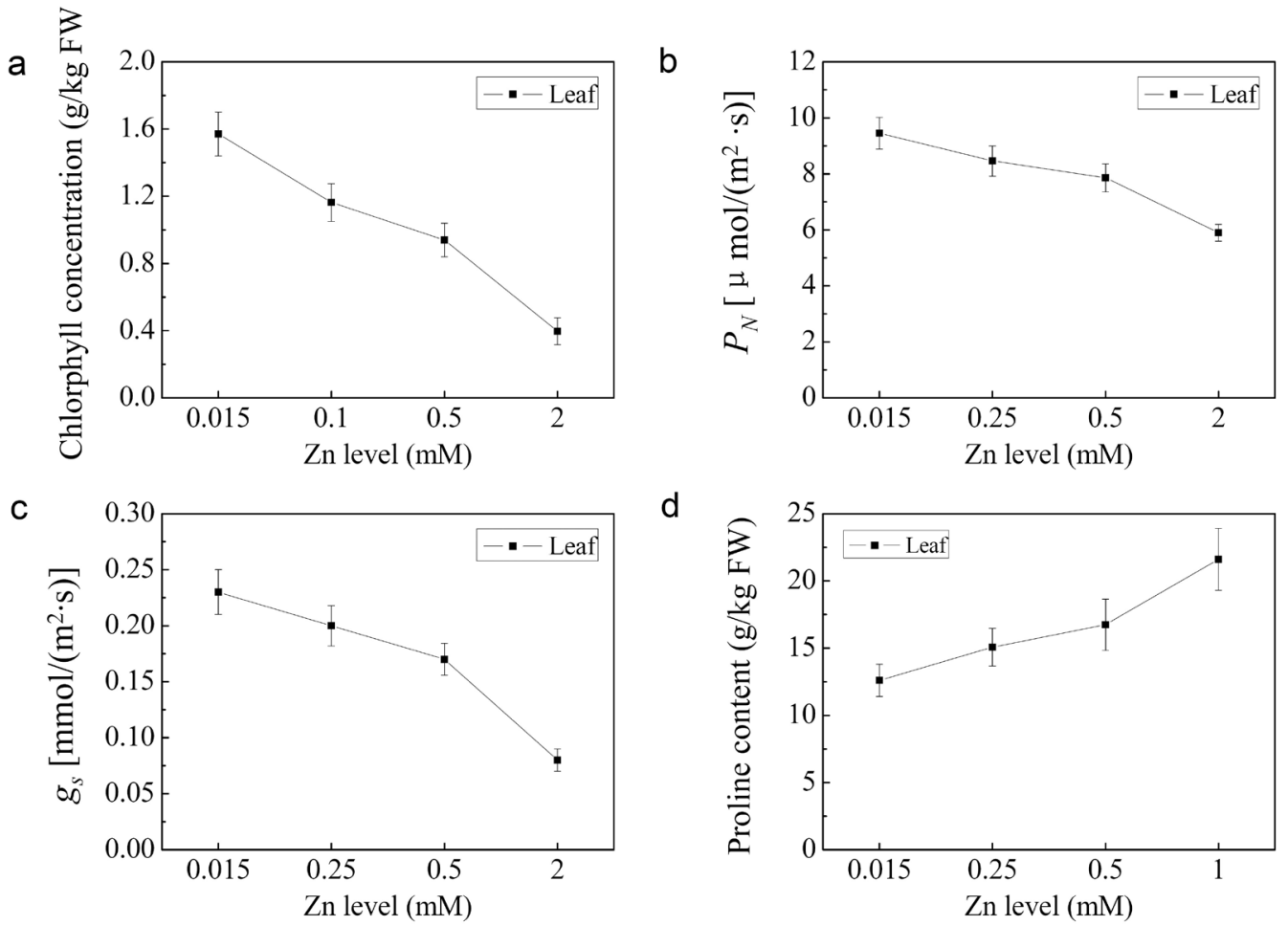

d
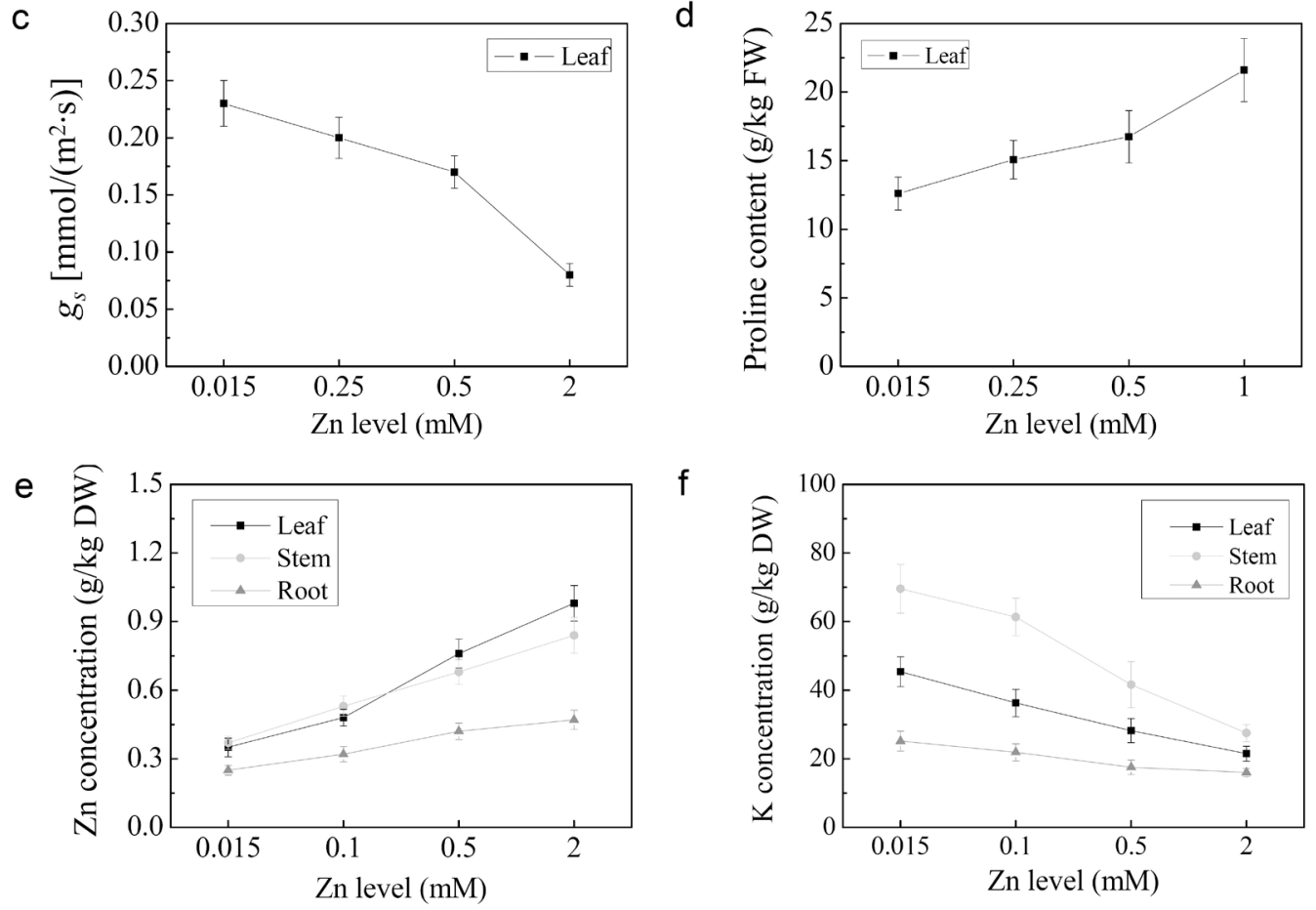

Figure 2. Biochemical analysis of peach seedlings in response to external zinc supply. Peach seedlings were grown in a $1 / 2 \mathrm{MS}$ solution supplemented with $0.015,0.1,0.5$, or $2 \mathrm{mM} \mathrm{ZnCl}_{2}$ for 21 days before analysis. a. Leaf chlorophyll concentration; b. photosynthetic rate $\left(P_{N}\right)$; c. stomatal conductance $\left(g_{s}\right)$; d. proline content; e. tissue zinc concentration; $\mathbf{f}$. tissue potassium concentration. The values are reported as means $\pm \mathrm{SE}(\mathrm{N}=24)$, calculated from 3 sets of experiments. 


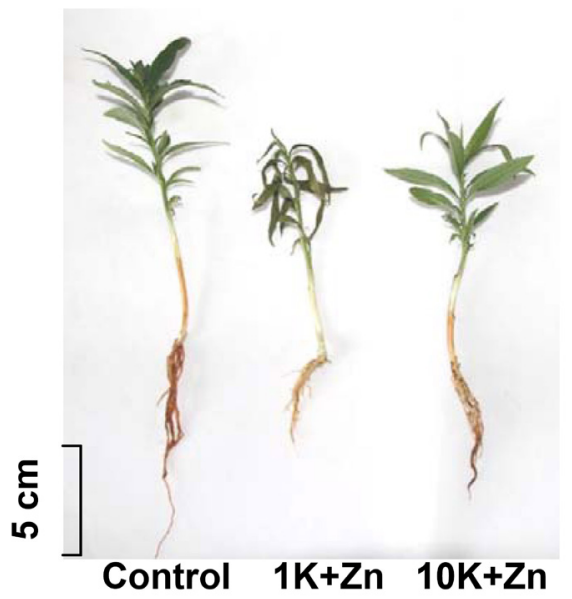

Figure 3. Morphology of peach seedlings under zinc toxicity and $\mathrm{K}^{+}$supply. Seedlings were treated for 10 days prior to analysis. $+\mathrm{Zn}$, means $2 \mathrm{mM} \mathrm{ZnCl}_{2}$ stress; 1 or $10 \mathrm{~K}$, indicates 1 or $10 \mathrm{mM} \mathrm{K}^{+}$.

Table 2. Peach seedling properties under two zinc treatment conditions and a control.

\begin{tabular}{lccc}
\hline Seedling property & \multicolumn{2}{c}{ Treatments } \\
\cline { 2 - 4 } & Control $(1 \mathrm{~K})$ & $1 \mathrm{~K}+\mathrm{Zn}$ & $10 \mathrm{~K}+\mathrm{Zn}$ \\
\hline Total fresh weight $(\mathrm{g})$ & $5.9 \pm 0.4^{* *}$ & $1.9 \pm 0.1$ & $3.6 \pm 0.3^{* *}$ \\
Total root length $(\mathrm{m})$ & $2.6 \pm 0.2^{* *}$ & $0.6 \pm 0.1$ & $1.3 \pm 0.1^{* *}$ \\
Total root surface area $\left(\mathrm{cm}^{2}\right)$ & $4.1 \pm 0.3^{* *}$ & $0.7 \pm 0.1$ & $1.9 \pm 0.1^{*}$ \\
Chlorophyll content $\left(\mathrm{g} / \mathrm{kg} \mathrm{FW}^{*}\right)$ & $1.6 \pm 0.1$ & $1.1 \pm 0.1$ & $1.4 \pm 0.1^{*}$ \\
$P_{N}\left[\mu \mathrm{mol} \cdot \mathrm{m}^{-2} \cdot \mathrm{s}\right]$ & $9.3 \pm 0.3$ & $5.9 \pm 0.2^{* *}$ & $8.2 \pm 0.4$ \\
$g_{s}\left[\mathrm{mmol} \cdot \mathrm{m}^{-2} \cdot \mathrm{s}\right]$ & $0.2 \pm 0.00$ & $0.1 \pm 0.02^{* *}$ & $0.2 \pm 0.01$ \\
Total K $\left.\mathrm{content}^{+} \mathrm{g} / \mathrm{kg} \mathrm{DW}\right)$ & $52.7 \pm 4.6^{* *}$ & $29.4 \pm 6.2$ & $36.7 \pm 6.3^{*}$ \\
Total $\mathrm{Zn}{ }^{2+}$ content $(\mathrm{g} / \mathrm{kg} \mathrm{DW})$ & $0.3 \pm 0.05$ & $0.7 \pm 0.08^{* *}$ & $0.4 \pm 0.06$ \\
Proline content $(\mathrm{g} / \mathrm{kg} \mathrm{FW})$ & $14.6 \pm 1.2^{*}$ & $11.2 \pm 0.9^{*}$ & $8.4 \pm 0.7$ \\
$\mathrm{H}_{2} \mathrm{O}_{2}$ content $(\mathrm{mmol} / \mathrm{kg} \mathrm{FW})$ & $0.2 \pm 0.04$ & $0.8 \pm 0.09^{* *}$ & $0.5 \pm 0.06^{* *}$ \\
\hline
\end{tabular}

Seedlings were subjected to treatments for 21 days prior to examination. $1 \mathrm{~K}+\mathrm{Zn}, 2 \mathrm{mM} \mathrm{K}^{+}$plus $2 \mathrm{mM} \mathrm{Zn}^{2+} ; 10 \mathrm{~K}+\mathrm{Zn}$, $10 \mathrm{mM} \mathrm{K}^{+}$plus $2 \mathrm{mM} \mathrm{Zn}^{2+} ; P_{N}$, photosynthetic rate; $g_{s}$, stomatal conductance. The values are reported as means \pm $\mathrm{SE}, \mathrm{N}=24$. *Significant difference among treatments $(0.01<* \mathrm{P}<0.05, * * \mathrm{P}<0.01$, independent samples $t$-test $)$.

\section{Escalated $\mathrm{K}^{+}$supply enhances activity of antioxidant enzymes under $\mathrm{Zn}$ toxicity}

Considering that the "Control+10K" treatment caused little effect to either growth status or physiological processes, including photosynthesis and antioxidant enzyme activities (SOD, POD, CAT, and APX), compared to the control (1 mM K ${ }^{+}$) treatment (Song ZZ, Guo $\mathrm{SL}$, Ma RJ and Yu ML, unpublished results), we carried out further studies focused on the application of normal $\mathrm{K}^{+}\left(1 \mathrm{mM} \mathrm{K}^{+}\right)$and escalated $\mathrm{K}^{+}\left(10 \mathrm{mM} \mathrm{K}^{+}\right)$supplies under high $\mathrm{Zn}$ toxicity, to illuminate whether external $\mathrm{K}^{+}$has any practical means in improving plant tolerance to $\mathrm{Zn}$ stress. Compared with the $1 \mathrm{~K}+\mathrm{Zn}\left(2 \mathrm{mM} \mathrm{Zn}\right.$ toxicity supplied with $\left.1 \mathrm{mM} \mathrm{K}^{+}\right)$treatment, elevated $\mathrm{K}^{+}$supply $(10 \mathrm{mM})$ significantly reduced endogenous $\mathrm{H}_{2} \mathrm{O}_{2}$ concentration, with approximately $38 \%$ loss (Table $2, \mathrm{P}<0.01, t$-test). Meanwhile, significantly higher activities of endogenous SOD, POD, and CAT were observed in the leaves of plants exposed to the $10 \mathrm{~K}+\mathrm{Zn}$ treatment, especially from day 15 to day 21 , compared with that of the $1 \mathrm{~K}+\mathrm{Zn}$ treat- 
ment (Figure 4a-c, $0.01<\mathrm{P}<0.05, t$-test). However, no significant difference in APX activity was detected between the additions of distinct $\mathrm{K}^{+}$supplies (Figure $4 \mathrm{~d}$ ).

a

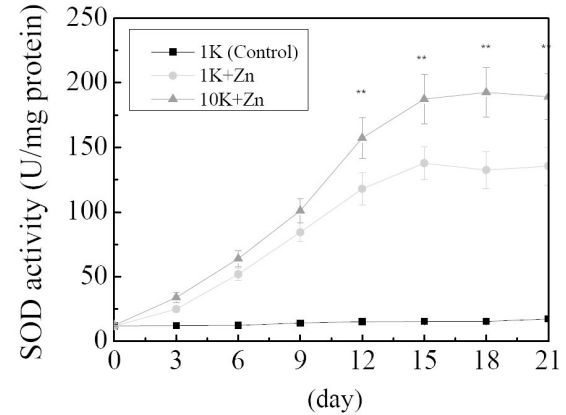

c

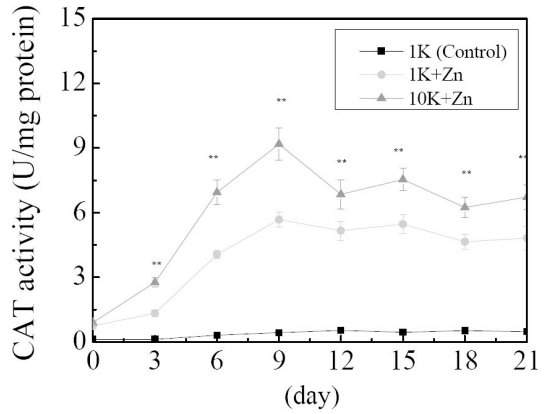

$\mathrm{b}$

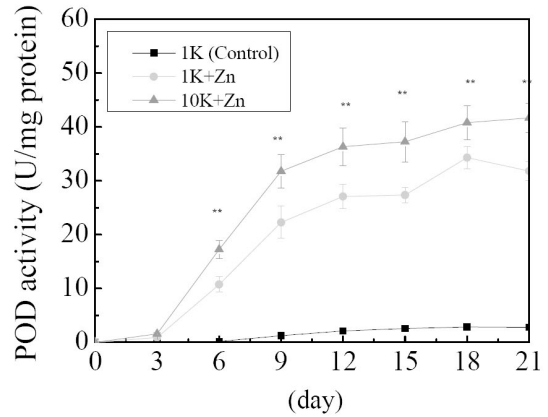

d

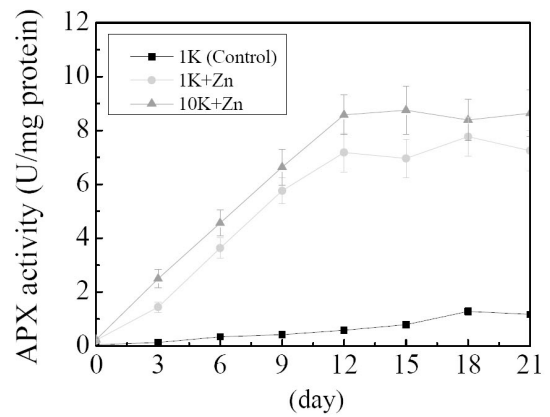

Figure 4. Activity of antioxidant enzymes in peach seedlings under zinc toxicity and $\mathrm{K}^{+}$supply. Seedlings were subjected to treatments for 21 days and leaves were collected for analysis at desired times. a. SOD activity; b. POD activity; c. CAT activity; d. APX activity. $+\mathrm{Zn}$, means $2 \mathrm{mM} \mathrm{ZnCl}_{2}$ stress; 1 or $10 \mathrm{~K}$, indicates 1 or $10 \mathrm{mM} \mathrm{K}^{+}$. The values are reported as means $\pm \mathrm{SE}(\mathrm{N}=24)$, calculated from 3 sets of experiments. *Statistical differences between seedlings grown under $1 \mathrm{mM} \mathrm{ZnCl}_{2}$ supplied with 1 and $10 \mathrm{mM} \mathrm{KCl}(* * \mathrm{P}<0.01$, independent samples $t$-test).

To elucidate the molecular mechanisms underlying the relationship between antioxidant enzyme activities and $\mathrm{Zn}$ toxicity, transcriptional profiles of putative antioxidation-related genes in peach leaves were analyzed in response to $\mathrm{Zn}$ toxicity. The genes encoding SOD, POD, and CAT had higher expression levels in peach leaves, with different dynamics, under the $10 \mathrm{~K}+\mathrm{Zn}$ treatment compared to the $1 \mathrm{~K}+\mathrm{Zn}$ treatment. In particular, expression levels of the SOD2, SOD3, $P O D 2, P O D 3$, and $C A T 1$ genes were significantly higher in peach leaves exposed to the $10 \mathrm{~K}+\mathrm{Zn}$ treatment than in leaves exposed to the $1 \mathrm{~K}+\mathrm{Zn}$ treatment (Figure 5, 0.01 $<\mathrm{P}<0.05, \mathrm{P}<0.01$, $t$-test), especially between days 15 to 21 . However, no obvious difference was observed in the transcript levels of the SOD1, SOD4, and PpeAPX genes (data not shown). In detail, expression of SOD2 and $P O D 2$ was slightly increased under the $1 \mathrm{~K}+\mathrm{Zn}$ treatment within the time points tested, but was dramatically enhanced in leaves under the $10 \mathrm{~K}+\mathrm{Zn}$ treatment (Figure $5 \mathrm{a}$ and $\mathrm{d}$, $0.01<\mathrm{P}<0.05, t$-test). Notably, expression of $S O D 3, P O D 3$, and $C A T 1$ was upregulated in plant leaves under the $1 \mathrm{~K}+\mathrm{Zn}$ treatment at the early stage but changed little after 15 days, whereas expression of these genes steadily increased in leaves after being exposed to the $10 \mathrm{~K}+\mathrm{Zn}$ treatment within the first 18 days (Figure 5b, e, and f, $\mathrm{P}<0.01$, $t$-test). Although $P O D 1$ expression steadily increased under both treatments, it was slightly higher in leaves under the $10 \mathrm{~K}+\mathrm{Zn}$ treatment than the $1 \mathrm{~K}+\mathrm{Zn}$ treatment at the several tested time points (Figure $5 \mathrm{c}$ ). 
a

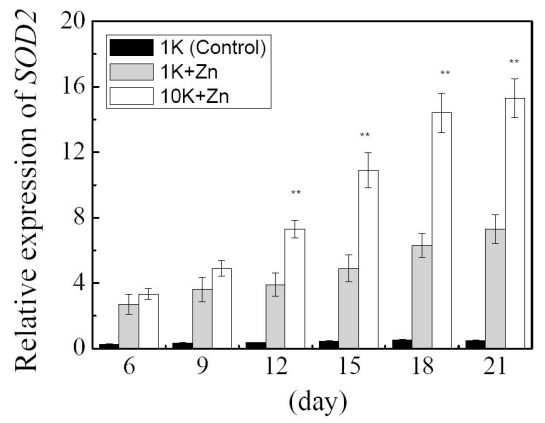

C

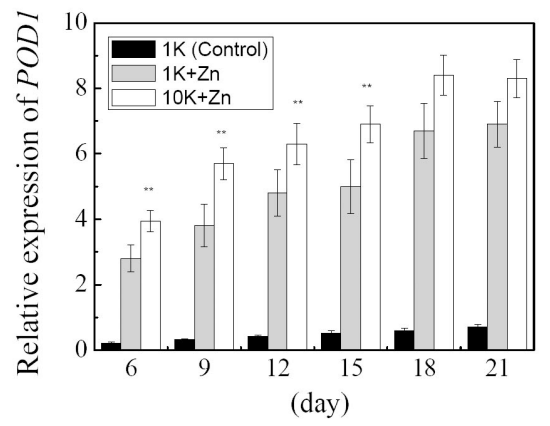

e

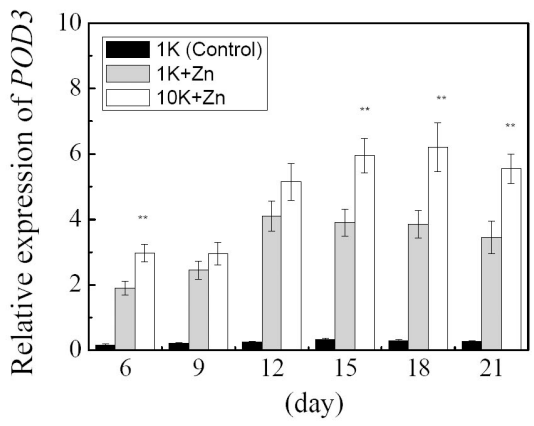

b

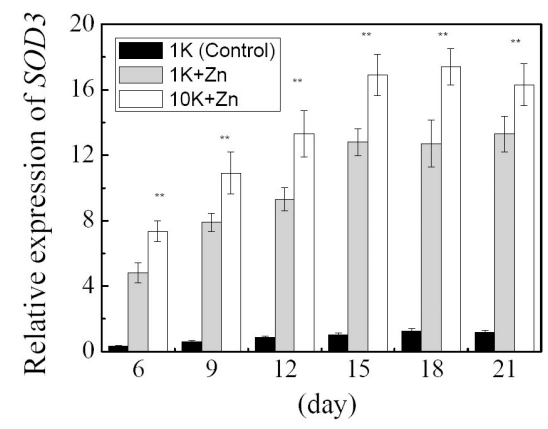

d

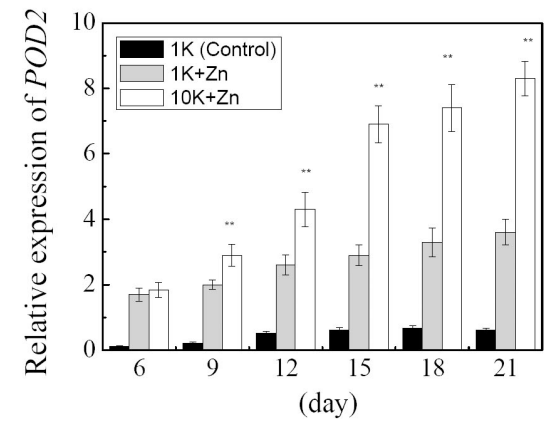

f

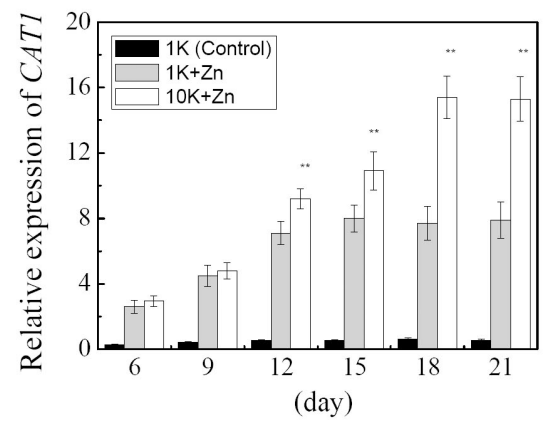

Figure 5. Relative expression of genes involved in the antioxidant defense system of peach seedlings under zinc toxicity. a. $S O D 2$, b. $S O D 3$, c. $P O D 1$, d. $P O D 2$, e. $P O D 3$, and f. $C A T 1$. All treatments were maintained for 21 days prior to analysis. Expression values are given as a ratio relative to the values of actin. $+\mathrm{Zn}$, means $2 \mathrm{mM} \mathrm{ZnCl}$ stress; 1 or $10 \mathrm{~K}$, indicates 1 or $10 \mathrm{mM} \mathrm{K}^{+}$. Data are reported as means of values obtained from four independent replicates $\pm \mathrm{SE}$. Statistical differences between seedlings grown under $1 \mathrm{mM} \mathrm{ZnCl}$ supplied with 1 and $10 \mathrm{mM} \mathrm{KCl}$ (** $<<0.01$, independent samples $t$-test).

\section{Expression profiles of the KT/HAK/KUP family genes in response to $\mathrm{Zn}$ toxicity}

Notably, Zn toxicity dramatically decreased tissue $\mathrm{K}^{+}$accumulation, while escalated $\mathrm{K}^{+}$supply $(10 \mathrm{~K}+\mathrm{Zn})$ practically restored tissue $\mathrm{K}^{+}$concentration under $\mathrm{Zn}$ toxicity (Table 2$)$. To further elucidate the molecular mechanisms underlying the relationship between $\mathrm{K}^{+}$acquisition and the $\mathrm{KT} / \mathrm{HAK} / \mathrm{KUP}$ family $\mathrm{K}^{+}$transporters, expression profiles of 16 putative $K U P$ genes in peach were evaluated in response to different $\mathrm{K}^{+}$supplies under $\mathrm{Zn}$ toxicity (for 21 days). Compared to control conditions, expression of seven, eight, and eight $K U P$ genes was 
significantly induced in leaf, stem and root tissues $(0.01<\mathrm{P}<0.05, \mathrm{P}<0.01, t$-test $)$, respectively, under $\mathrm{Zn}$ toxicity $(1 \mathrm{~K}+\mathrm{Zn})$, whereas transcript levels of three genes (i.e., KUP3, KUP4, and KUP6) in leaf and four genes (including KUP1, KUP5, KUP6, and KUP11) in stem were reduced $(0.01<\mathrm{P}<0.05, \mathrm{P}<0.01, t$-test $)$. Expression of $K U P 15$ changed little, either under $1 \mathrm{~K}+\mathrm{Zn}$ or $10 \mathrm{~K}+\mathrm{Zn}$ treatment (Figure 6 ). Together, the differential response of these genes may intricately contribute to the reduced tissue $\mathrm{K}^{+}$concentration under $\mathrm{Zn}$ toxicity. Compared to control conditions, elevated $\mathrm{K}^{+}$supply $(10 \mathrm{~K}+\mathrm{Zn})$ significantly enhanced expression of eleven, ten, and ten $K U P$ genes in leaf, stem and root tissues respectively, under $\mathrm{Zn}$ toxicity (Figure 6, $0.01<\mathrm{P}<0.05, \mathrm{P}<0.01, t$-test), which may partially explain the increased $\mathrm{K}^{+}$accumulation in tested organs under $\mathrm{Zn}$ toxicity (Table 2 ).

a

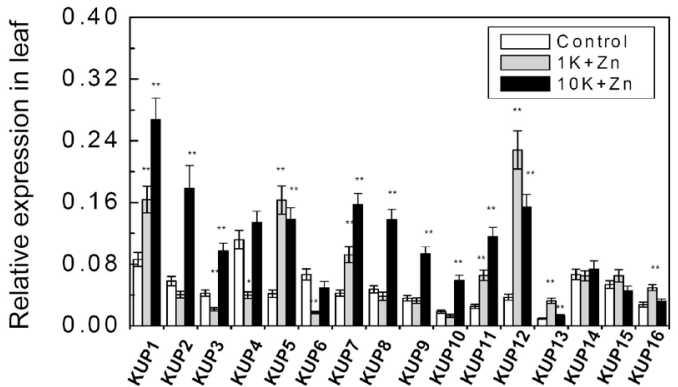

b

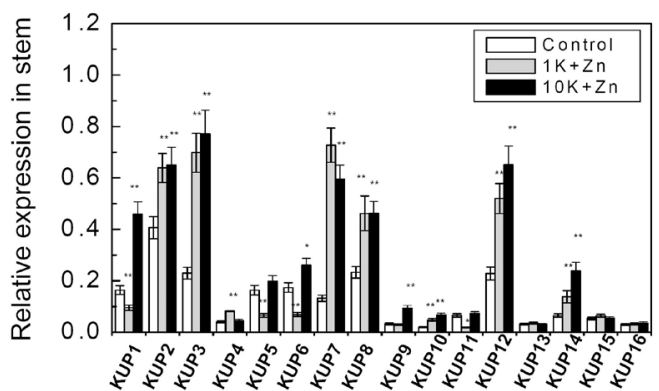

c

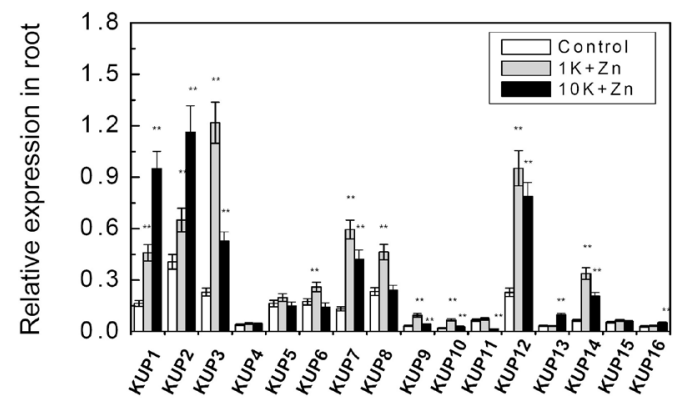

Figure 6. Relative expression of PpeKUP genes under zinc toxicity. All treatments were maintained for 21 days prior to analysis. Expression values are given as a ratio relative to the values of actin. $+\mathrm{Zn}$, means $2 \mathrm{mM} \mathrm{ZnCl}_{2}$ stress; 1 or $10 \mathrm{~K}$, indicates 1 or $10 \mathrm{mM} \mathrm{K}$. The values are reported as means $\pm \mathrm{SE}(\mathrm{N}=24)$, calculated from 3 sets of experiments. *Statistical differences between seedlings grown under control and $\mathrm{ZnCl}_{2}$ stresses $(* * \mathrm{P}<0.01$, independent samples $t$-test). 


\section{DISCUSSION}

As an essential microelement involved in numerous physiological processes, trace amounts of $\mathrm{Zn}$ application contribute to fruit quality in particular (Zhang et al., 2013; Saadati et al., 2013). However, excessive amounts of $\mathrm{Zn}$ can lead to toxicity symptoms in plants. In this present study, peach seedlings exhibited better tolerance to heavy $\mathrm{Zn}$ toxicity than several annual plants, including maize, rice, and Arabidopsis. Notably, peach as a perennial woody plant contains more lignin than these annual plants, which may partially explain the improved tolerance to heavy metals. It is also worth mentioning that the $\mathrm{Zn}^{2+}$ concentration in shoots was simultaneously reinforced with increasing $\mathrm{Zn}^{2+}$ supplies, implying that peach 'Yoshihime' seedlings may have an excellent capacity for $\mathrm{Zn}^{2+}$ accumulation, and excess $\mathrm{Zn}^{2+}$ may be further transported or deposited to the aboveground parts of the seedlings. Nonetheless, our findings imply that peach 'Yoshihime' seedlings may have higher $\mathrm{Zn}$ tolerance and $\mathrm{Zn}$ accumulation capacities than normal crops

Heavy $\mathrm{Zn}$ toxicity has been reported to reduce plant growth by causing internal physiological changes (Kozlov, 2004; Street et al., 2007). Indeed, Zn toxicity (2 mM) resulted in severe growth damage and affected biochemical processes, especially photosynthetic performance, in peach seedlings in the current study. Interestingly, elevated $\mathrm{K}^{+}$supply $\left(10 \mathrm{mM} \mathrm{K}^{+}\right)$ beneficially abolished the damage caused by $\mathrm{Zn}$ toxicity, and endowed peach seedlings with dramatically strengthened photosynthetic performance, evidenced by enhanced total chlorophyll concentration, $g_{s}$ and $P_{N}$, which may directly explain their enhanced growth performance and increased number of green leaves.

Notably, elevated $\mathrm{K}^{+}$supply reduced the internal $\mathrm{H}_{2} \mathrm{O}_{2}$ concentration, suggesting an enhanced capacity for protection from oxidative damage by Zn toxicity. Simultaneously, significantly enhanced activities of endogenous SOD, POD, and CAT in peach leaves were observed under the $10 \mathrm{~K}+\mathrm{Zn}$ treatment. Correspondingly, the transcripts of relative genes involved in plant antioxidant systems were significantly increased in the leaves of peaches under test. In particular, proline is an important stress-related metabolite that contributes to osmotic adjustment and enzyme stabilization, enabling plants to better tolerate adverse abiotic stresses (Choudhary et al., 2007; Huang et al., 2013). Under Zn toxicity, elevated $\mathrm{K}^{+}$supply (10 mM $\mathrm{K}^{+}$) significantly reduced leaf proline concentrations, which may partially support the theory that proline contributes to plant performance and tolerance under $\mathrm{Zn}$ toxicity. We speculate that escalated $\mathrm{K}^{+}$supply favorably contributes to plant antioxidant systems by enhancing ROSscavenging capacity for oxidative damage protection, which further provides peach seedlings with better performance under $\mathrm{Zn}$ toxicity.

$\mathrm{K}^{+}$beneficially contributes to plant tolerance to various abiotic stresses, such as drought, salt, and cold (Rai et al., 2008; Mian et al., 2011; Li et al., 2011; Song and Su, 2013; Song et al., 2014a, 2015). Zn toxicity dramatically reduces many metabolic processes in plants, including $\mathrm{K}^{+}$nutrition and homeostasis. To cope with such adverse toxicity stress, plants may have to improve their internal nutritional status or strengthen metabolic systems to maintain basic growth. Notably, expression of 15 out of $16 \mathrm{KUP}$ genes was greatly increased in individual tissues under $\mathrm{Zn}$ toxicity stress, directly indicating that $\mathrm{K}^{+}$acquisition and transport systems were enhanced, which possibly mobilized the 'luxury uptake' of external $\mathrm{K}^{+}$via $\mathrm{K}^{+}$ transporters to protect $\mathrm{K}^{+}$-dependent cellular processes and maintain basic growth. Moreover, elevated $\mathrm{K}^{+}$supply beneficially strengthened peach root formation under $\mathrm{Zn}$ stress, which possibly ensured powerful $\mathrm{K}^{+}$uptake via plant roots when exposed to sufficient $\mathrm{K}^{+}$supply. Si- 
multaneously, all of the KUP genes, except $K U P 4$ and $K U P 15$, were significantly upregulated in individual tissues under $\mathrm{Zn}$ toxicity after supply of plentiful amounts of $\mathrm{K}^{+}$, indicating that enhanced expression of $\mathrm{K}^{+}$transporter genes surely guarantees $\mathrm{K}^{+}$uptake, which is then sufficiently transported or dispatched into the aboveground components, further protecting $\mathrm{K}^{+}$-dependent metabolic processes, including photosynthesis. Indeed, elevated $\mathrm{K}^{+}$supply favorably increased tissue $\mathrm{K}^{+}$accumulation under $\mathrm{Zn}$ toxicity. Escalated $\mathrm{K}^{+}$supply definitely favorably enhanced the expression of genes involved in plant antioxidant systems, which better explains the corresponding strengthened activity of antioxidant enzymes (Figure 4). Nonetheless, these findings directly suggest that escalated $\mathrm{K}^{+}$supply contributes to enhancing plant antioxidant systems, which may partially explain their improved performance under $\mathrm{Zn}$ toxicity.

Although the molecular basis of KT/HAK/KUP family transporters is largely unclear in peach, these transporters were closely involved in $\mathrm{K}^{+}$nutrition and homeostasis, especially under $\mathrm{Zn}$ toxicity, further protecting plant photosynthetic performance and the antioxidative defense system. Nonetheless, our findings convincingly support the key role of $\mathrm{K}^{+}$in improving plant tolerance to abiotic stresses.

\section{Conflicts of interest}

The authors declare no conflict of interest.

\section{ACKNOWLEDGMENTS}

Research supported by the Jiangsu Independent Innovation of Agricultural Science [\#CX(13)3049], the China Postdoctoral Scientific Research Foundation (\#2015M571713), the Jiangsu Agriculture Science and Technology Innovation Fund [\#CX(14)2015], and the Natural Science Foundation of Jiangsu Province (\#BK20140755).

\section{REFERENCES}

Alemán F, Nieves-Cordones M, Martínez V and Rubio F (2011). Root $\mathrm{K}\left({ }^{+}\right)$acquisition in plants: the Arabidopsis thaliana model. Plant Cell Physiol. 52: 1603-1612.

Barak P and Helmke PA (1993). The chemistry of zinc. In: Zinc in soils and plants (Robson AD, ed.). Developments in Plants and Soil Sciences, Kluwer Academic Press, New York, 1-13.

Bradford MM (1976). A rapid and sensitive method for the quantitation of microgram quantities of protein utilizing the principle of protein dye binding. Anal. Biochem. 72: 248-254.

Cambrollé J, Mancilla-Leytón JM, Muñoz-Vallés S, Luque T, et al. (2012). Zinc tolerance and accumulation in the saltmarsh shrub Halimione portulacoides. Chemosphere 86: 867-874.

Choudhary M, Jetley UK, Abash Khan M, Zutshi S, et al. (2007). Effect of heavy metal stress on proline, malondialdehyde, and superoxide dismutase activity in the cyanobacterium Spirulina platensis-S5. Ecotox. Environ. Safe 66: 204-209.

Coskun D, Britto DT, Li M, Oh S, et al. (2013). Capacity and plasticity of potassium channels and high-affinity transporters in roots of barley and Arabidopsis. Plant Physiol. 162: 496-511.

Davies C, Shin R, Liu W, Thomas MR, et al. (2006). Transporters expressed during grape berry (Vitis vinifera L.) development are associated with an increase in berry size and berry potassium accumulation. J. Exp. Bot. 57: 3209-3216.

Demiral MA and Köseoglu AT (2005). Effect of potassium on yield, fruit quality, and chemical composition of greenhousegrown galia melon. J. Plant Nutr. 28: 93-100.

Ernst WHO, Nelissen HJM and Ten Bookum WM (2000). Combination toxicology of metal-enriched soils: physiological responses of a $\mathrm{Zn}$ - and Cd-resistant ecotype of Silene vulgaris on polymetallic soils. Environ. Exp. Bot. 43: 55-71.

Foyer CH and Noctor G (2005). Oxidant and antioxidant signalling in plants: a re-evaluation of the concept of oxidative stress in a physiological context. Plant Cell Environ. 28: 1056-1071. 
Grabov A (2007). Plant KT/KUP/HAK potassium transporters: single family-multiple functions. Ann. Bot. 99: 1035-1041. Hartz TK, Johnstone PR, Francis DM and Miyao EM (2005). Processing tomato yield and fruit quality improved with potassium fertigation. HortScience 40: 1862-1867.

Huang XS, Wang W, Zhang Q and Liu JH (2013). A basic helix-loop-helix transcription factor, PtrbHLH, of Poncirus trifoliata confers cold tolerance and modulates peroxidase-mediated scavenging of hydrogen peroxide. Plant Physiol. 162: 1178-1194.

Kabata-Pendias A and Pendias H (2001). Trace elements in soils and plants. 3rd edn. CRC Press, Boca Ratón, Florida.

Kozlov MV (2004). Retrospective analysis of the age at death in two heavily polluted and two unpolluted Russian towns. Chemosphere 56: 405-410.

Kumar N, Kumar S, Vats SK and Ahuja PS (2006). Effect of altitude on the primary products of photosynthesis and the associated enzymes in barley and wheat. Photosynth. Res. 88: 63-71.

Li M, Li Y, Li H and Wu G (2011). Overexpression of AtNHX5 improves tolerance to both salt and drought stress in Broussonetia papyrifera (L.) Vent. Tree Physiol. 31: 349-357.

Lombardi L and Sebastiani L (2005). Copper toxicity in Prunus cerasifera: growth and antioxidant enzymes responses of in vitro grown plants. Plant Sci. 168: 797-802.

Maathuis FJM and Sanders D (1996). Mechanisms of potassium absorption by higher plant roots. Physiol. Plant 96: 158168.

Mian A, Oomen RJ, Isayenkov S, Sentenac $\mathrm{H}$, et al. (2011). Over-expression of an $\mathrm{Na}^{+}$- and $\mathrm{K}^{+}$-permeable $\mathrm{HKT}$ transporter in barley improves salt tolerance. Plant J. 68: 468-479.

Morillo J, Usero J and Gracia I (2004). Heavy metal distribution in marine sediments from the southwest coast of Spain. Chemosphere 55: 431-442.

Murashige T and Skoog F (1962). A revised medium for rapid growth and bioassays with tobacco tissue cultures. Physiol. Plant 15: 473-497.

Rai RK, Singh P, Shrivastava AK and Suman A (2008). Modulation of low-temperature induced biochemical changes in bud and root band zones of sugar cane sets by potassium, zinc, and ethrel for improving sprouting. J. Agric. Food Chem. 56: 11976-11982.

Saadati S, Moallemi N, Mortazavi SMH and Seyyednejad SM (2013). Effects of zinc and boron foliar application on soluble carbohydrate and oil contents of three olive cultivars during fruit ripening. Sci. Hortic. 164: 30-34.

Santa-Maria GE, Rubio F, Dubcovsky J and Rodriguez-Navarro A (1997). The HAK1 gene of barley is a member of a large gene family and encodes a high-affinity potassium transporter. Plant Cell 9: 2281-2289.

Singh AK, Kumar R, Pareek A, Sopory SK, et al. (2012). Overexpression of rice CBS domain containing protein improves salinity, oxidative, and heavy metal tolerance in transgenic tobacco. Mol. Biotechnol. 52: 205-216.

Song ZZ and Su YH (2013). Distinctive potassium-accumulation capability of alligatorweed (Alternanthera philoxeroides) links to high-affinity potassium transport facilitated by $\mathrm{K}^{+}$-uptake systems. Weed Sci. 61: 77-84.

Song ZZ, Yang SY, Zuo J and Su YH (2014a). Over-expression of ApKUP3 enhances potassium nutrition and drought tolerance in transgenic rice. Biol. Plant 58: 649-658.

Song ZZ, Yang SY, Zhu H, Jin M, et al. (2014b). Heterologous expression of an alligatorweed high-affinity potassium transporter gene enhances salinity tolerance in Arabidopsis thaliana. Am. J. Bot. 101: 840-850.

Song ZZ, Ma RJ and Yu ML (2015). Genome-wide analysis and identification of KT/HAK/KUP potassium transporter gene family in peach (Prunus persica). Genet. Mol. Res. 14: 774-787.

Street RA, Kulkarni MG, Stirk WA, Southway C, et al. (2007). Toxicity of metal elements on germination and seedling growth of widely used medicinal plants belonging to Hyacinthaceae. Bull. Environ. Contam. Toxicol. 79: 371-376.

Turchi A, Tamantini I, Camussi AM and Racchi ML (2012). Expression of a metallothionein A1 gene of Pisum sativum in white poplar enhances tolerance and accumulation of zinc and copper. Plant Sci. 183: 50-56.

Véry AA and Sentenac H (2003). Molecular mechanisms and regulation of $\mathrm{K}^{+}$transport in higher plants. Annu. Rev. Plant Biol. 54: 575-603.

Wang WB, Kim YH, Lee HS, Kim KY, et al. (2009). Analysis of antioxidant enzyme activity during germination of alfalfa under salt and drought stresses. Plant Physiol. Biochem. 47: 570-577.

Worthington V (2001). Nutritional quality of organic versus conventional fruits, vegetables, and grains. J. Altern. Complement. Med. 7: 161-173.

Zhang Y, Fu C, Yan Y, Wang YA, et al. (2013). Zinc sulfate and sugar alcohol zinc sprays at critical stages to improve apple fruit quality. HortTechnology 23: 490-497. 